\title{
A posited fuzzy-evolutionary computational model to minimise the tracking error of an option-replicating portfolio
}

\author{
Mohammad Khoshnevisan, Edgars Vimba and Sukanto Bhattacharya* \\ *Assistant Professor of Finance, Department of International Business and Management, \\ Dickinson College, PO Box 1773, Carlisle, PA 17013, USA. \\ Tel: + 1717254 8931,Fax: + 1717245 1025, E-mail: bhattacs@dickinson.edu
}

Received (in revised form): 21st May, 2007

\begin{abstract}
Mohammad Khoshnevisan received his PhD degree specialising in computational finance from the University of Melbourne, Australia. In his doctoral thesis he developed an optimal control model for borrowing in the Australian petroleum industry. He has published a vast number of articles in international journals, conference proceedings and refereed monographs and many of his works are cited by elite academic/research organisations such as the American Mathematical Society and the International Statistical Institute. He is a senior lecturer in Finance at Griffith University, Australia.
\end{abstract}

Edgars Vimba is presently a undergraduate student in the Department of Economics, Judd A. and Marjorie Weinberg College of Arts and Sciences at Northwestern University. He is double majoring in Economics and Mathematics. Edgars has been a member of the Sigma Beta Delta International Honor Society in Business, Management and Administration since 2006 and has won the prestigious President's Foreign Student Scholarship (2004-2007) at Alaska Pacific University, USA.

Sukanto Bhattacharya received his PhD degree in Information Technology from Bond University, Australia in 2004. $\mathrm{He}$ is an internationally recognised authority on fuzzy and neutrosophic optimal control models and their applications in financial risk management. He has many publications in international journals, conference proceedings, refereed monographs as well as a book chapter. His publications encompass a varied range of disciplines ranging from forensic accounting to quantum metaphysics. He is an assistant professor of Finance in the Department of International Business and Management at Dickinson College, USA.

\section{Practical applications}

This article builds on an earlier work that used a Genetic Algorithm approach to computationally demonstrate the evolutionary optimality of the Black-Scholes function. We have extended the same computational scheme to posit a practically usable portfolio optimisation tool that could effectively minimise the cumulative hedging error of a replicating portfolio for a multi-asset, best-of option.

\section{Abstract}

In this paper, we build on an earlier work using a haploid genetic algorithm (hGA) model that demonstrated the evolutionary optimality of the Black-Scholes options pricing functional form. We adopt the same hGA model in a similar problem context and posit adding on a fuzzy logic controller to come up with a practically usable portfolio management decision support tool that could minimise the cumulative hedging error. As the optimality constraints are dependent on abruptly changing and often ambiguous market conditions, it provides a fertile ground for applying such fuzzy optimisation. Journal of Derivatives \& Hedge Funds (2007) 13, 214-219. doi:10.1057/palgrave.jdhf.1850075
Journal of Derivatives \& Hedge Funds, Vol. 13 No. 3, 2007 pp. 214-219 (C) 2007 Palgrave Macmillan Ltd $1753-9641 \$ 30.00$ 
Keywords: haploid genetic algorithm; replicating portfolio; fuzzy logic controller

\section{INTRODUCTION}

A financial-structured product often takes the form of a complex, multi-asset option whereby an investor receives a terminal payoff that is tied to the price or performance of any one of the several assets enveloped within the structure. The premium to be paid by the investor is determined by means of a pricing formula akin to the Black-Scholes expected-payoff formulation but in a multi-dimensional form. ${ }^{1}$ However, the actual utility from the financial-structured product at the end of the investment horizon depends on how well the assets within the structure have been 'managed' during the lockin period to adapt to the forever-changing asset market conditions. $^{2}$

For example, if the financial-structured product is actually a multi-asset, best-of option whose payoff is linked to the best-performing asset enveloped within that structure, then the payoff from this option can be replicated using a portfolio consisting of investments in the various constituent assets along with an investment in a nearly risk-free asset like a commercial bank fixed-deposit (if backed by a governmental authority like the FDIC in the US) or a government treasury bill. The proportion of invested funds in each of the risky assets as well as the risk-free asset is determined according to a rather complex allocation formula, which has to be re-calculated periodically to ensure that it is replicated with minimum error.

\section{PROBLEM FORMULATION}

Let a financial-structured product be made up of an envelope of $J$ different assets such that the investor has the right to claim the return on the best-performing asset out of that envelope after a stipulated lock-in period. Then, if one of the $J$ assets in the envelope is the risk-free asset, then the investor gets assured of a minimum return equal to the risk-free rate $i$ on his invested capital at the termination of the stipulated lock-in period. This effectively means that his or her investment becomes endogenously capital-guaranteed as the terminal wealth, even at its worst, cannot be lower in value to the initial wealth plus the return earned on the risk-free asset minus a finite cost of portfolio insurance, which is paid as the premium to the option writer.

Then, the expected present value of the terminal option payoff is obtained as follows:

$$
\begin{aligned}
\hat{E}(r)_{t=T}= & \operatorname{Max}\left[w, \operatorname{Max}_{j}\left\{\mathrm{e}^{-i t} E\left(r_{j}\right)_{t=T}\right\}\right], \\
& j=1,2 \ldots J-1
\end{aligned}
$$

In the above equation, $i$ is the rate of return on the risk-free asset and $T$ is the length of the investment horizon in continuous time and $w$ is the initial wealth invested, that is ignoring insurance cost, if the risk-free asset outperforms all other assets, then we obtain:

$$
\hat{E}(r)_{t=T}=w \mathrm{e}^{i T} / \mathrm{e}^{i T}=w
$$

Now what is the probability of each of the $(J-1)$ risky assets performing worse than the risk-free asset? Even if we assume that there are some cross-correlations present among the $(J-1)$ risky assets, given the statistical nature of the risk-return trade-off, the joint probability of all these assets performing worse than the riskfree asset will be very low over even moderately long investment horizons. This probability will keep going down with every additional risky asset added to the envelope. Thus, this 
probability can become quite negligible if we consider sufficiently large values of $n$. In this paper, however, we have taken $J=3$ mainly for computational simplicity, as closed-form pricing formulations become extremely difficult to obtain with $J>3$.

For an option writer who is looking to hedge his or her position, the expected utility maximisation criterion will require the tracking error to be at a minimum at each point of rebalancing, where the tracking error is the difference between the expected payoff on the best-of option and the replicating portfolio value at that point. Then, given a necessarily biological basis of the evolution of utility forms, a haploid genetic algorithm ( $h \mathrm{GA}$ ) model, which, as a matter of fact, can be shown to be statistically equivalent to multiple multi-armed bandit processes, was shown to show satisfactory convergence with the Black-Scholes-type expected utility formulation to the problem in Bhattacharya and Kumar. ${ }^{3}$ The underlying premise of their study was essentially similar to that adopted in case of establishing the biological basis of an expected utility function by Robson ${ }^{4,5}$ and Becker. ${ }^{6}$ Bhattacharya and Kumar $^{3}$ actually computationally validated the evolutionary optimality of the Black-Scholes function over all other possible functions within the same domain.

At each point of re-balancing, the tracking error has to be minimised if the difference between the expected option payoff and the replicating portfolio value is to be minimised. The more significant this difference, the more will be the cost of re-balancing associated with correcting the tracking error, and as these costs cumulate, the less will be the ultimate utility of the hedge to the option writer at the end of the lock-in period. The cumulative tracking error $\xi$ over the lock-in period is given as:

$$
\xi=\sum_{t}\left|E(r)_{t}-v_{t}\right|
$$

Here, $E(r)_{t}$ is the expected best-of option payoff at time-point $t$ and $v_{t}$ is the replicating portfolio value at that point of time. Then, the replicating portfolio value at time $t$ is obtained as the following linear form:

$$
\begin{gathered}
v_{t}=\left(p_{0}\right)_{t} \mathrm{e}^{i t}+\sum_{j}\left\{\left(P_{j}\right)_{t}\left(S_{j}\right)_{t}\right\}, \\
j=1,2 \ldots J-1
\end{gathered}
$$

Here, $\left(S_{j}\right)_{t}$ is the realised return on asset $j$ at timepoint $t$ and $p_{1}, p_{2} \ldots p_{J-1}$ are the respective allocation proportions of investment funds among the $J-1$ risky assets at time-point $t$ and $\left(p_{0}\right)_{t}$ is the allocation for the risk-free asset at time-point $t$. Of course:

$$
\left(P_{0}\right)_{t}=1-\sum_{j}\left(P_{j}\right)_{t}
$$

It is the portfolio weights, that is, the $p_{0}$ and $p_{j}$ values that are of critical importance in determining the size of the tracking error. The correct selection of these portfolio weights will ensure that the replicating portfolio accurately tracks the option. The option value on the best of two risky assets plus one risk-free asset is derived according to the standard Black-Scholes-type formulation. ${ }^{7}$

The terminal payoff from such a financialstructured product would be that on the asset ending up as the best performer among the three assets within the envelope, that is

$$
r_{t=T}=\operatorname{Max}\left(S_{0}, S_{1}, S_{2}\right)_{t=T}
$$

Two assets with realised returns $S_{1}$ and $S_{2}$ can be considered risky, that is, $\sigma_{S_{1}}^{2}, \sigma_{S_{2}}^{2}>0$, while $S_{0}$ may be considered risk-free, for example the return on a government treasury bill, that is $\sigma_{S_{0}}^{2} \approx 0$. Then, a dynamic hedging scheme for the issuer of this financial-structured product; that is 
the option writer, would be to invest in a replicating portfolio consisting of the three assets, with funds allocated in particular proportions in accordance with the objective of maximising expected utility, that is minimising the tracking error. Then, the replicating portfolio at $t$ for our three-asset financialstructured product is:

$$
v_{t}=\left(p_{0}\right)_{t} \mathrm{e}^{i t}+\left(p_{1}\right)_{t}\left(S_{1}\right)_{t}+\left(p_{2}\right)_{t}\left(S_{2}\right)_{t}
$$

Then, the tracking error at time-point $t$ is given as the difference between the payoff on the option at time-point $t$ and the value of the replicating portfolio at that time:

$$
\begin{aligned}
\left|\varepsilon_{t}\right|= & \left|\operatorname{Max}\left(S_{0}, S_{1}, S_{2}\right)_{t}-V_{t}\right| \text { ie } \\
\left|\varepsilon_{t}\right|= & \mid \operatorname{Max}\left(S_{0}, S_{1}, S_{2}\right)_{t}-\left\{\left(p_{0}\right)_{t} \mathrm{e}^{i t}+\left(p_{1}\right)_{t}\left(S_{1}\right)_{t}\right. \\
& \left.+\left(P_{2}\right)_{t}\left(S_{2}\right)_{t}\right\} \mid
\end{aligned}
$$

If $\left|\varepsilon_{t}\right| \approx 0$, then the option writer is perfectly hedged at time-point $t$. If $\left|\varepsilon_{t}\right| \approx 0$ can be maintained for the entire lock-in period, then one can say that the dynamic hedging scheme has worked perfectly, resulting in utility maximisation for the option writer.

Bhattacharya and Kumar ${ }^{3}$ developed and computationally implemented a simple $h \mathrm{GA}$ that demonstrated that the optimal hedge ratio that emerges out of the succeeding generations as the dominant phenotype indeed converges to that dictated by a multi-dimensional version of Black-Scholes model.

Bhattacharya and $\mathrm{Kumar}^{3}$ carried out their work with primarily a broader, philosophical purpose of demonstrating the biological superiority of a dynamic hedging parameter based on the multi-dimensional Black-Scholes model. We recognise, however, that their computational approach does have an innate attractiveness to be considered as a decision support tool in the context of real-time portfolio management decision making.

However, the actual solving of the stochastic optimisation problem of minimising the option tracking error in real time is found to be particularly susceptible to getting stuck in local optima especially when the return on the risky assets underlying the best-of option have temporally unstable correlation and volatility. Such instability gives rise to linguistic ambiguity in setting the target function as well as the constraints and renders the model untenable as a practical decision support tool.

We propose that this issue is best addressed by incorporation of a fuzzy logic component into the $h \mathrm{GA}$ model for practical considerations.

\section{INCORPORATING A SIMPLE FUZZY LOGIC CONTROLLER (FLC) INTO THE $h$ GA MODEL}

Evolutionary optimisation algorithms like an $h \mathrm{GA}$ principally work by trying to optimally prioritise between exploitation (existing knowledge) and exploration (new knowledge). One of the primary goals in an evolutionary optimisation set-up is to avoid getting stuck in local optima (premature convergence) in the effort to optimally prioritise between exploitation and exploration. As the knowledge base (existing as well as new) often consists of vague and imprecise information, the $h \mathrm{GA}$ performance can be better controlled using fuzzy logic controllers (FLCs). The FLC control process allows for an ideal man-machine interface for the optimal prioritisation between exploitation and exploration. Especially for many of the longer term options, implied volatility measures are often based on subjective and imprecise probability assessments that can 
significantly slow down the search speed. ${ }^{8}$ In general, $h$ GAs controlled by FLCs can resolve the imprecise information dynamically during run-time, thereby allowing faster adaptation. ${ }^{9}$

The principal goal is to use an FLC with an input that is any combination of the genetic algorithm performance measures, for example, in our multi-asset dynamic hedging problem, it could very well be the mean square tracking error of the replicating portfolio. In a feedback control mechanism, the current performance measures of the genetic algorithm are routed through the FLC, which computes new control parameter values that will be subsequently fed into the genetic algorithm. Standard genotype diversity measures like Hamming Distance, Euclidian Distance and entropy measures are also possible input candidates for the FLC along with common phenotype diversity measures like the span measure where:

$$
\operatorname{span}=\left[1 /(N-1) \sum\left(f_{i}-f^{*}\right) 2\right]^{1 / 2}\left[1 / N \sum f_{i}\right]^{-1}
$$

The above span formula applies to any predefined fitness criteria $f_{i}$ and the mean fitness criterion $f^{*}$ for an $N$-asset replicating portfolio.

Let us follow the simple rule-based logic as used in Bhattacharya and Kumar, ${ }^{3}$ that is, at each periodic re-balancing point $k$ per cent of funds are allocated to the observed best-performing risky asset and the balance $(90-k)$ per cent to the other risky asset so as to keep the portfolio self-financing after the initial investment. In the context of this three-asset portfolio dynamic hedging problem, a simple fuzzy rule pseudo code could then be implemented as follows:

IF

$$
\left[r_{i}-\operatorname{MAX}\left(r_{0}, r_{1}, r_{2}\right)\right]^{2} \text { is small }
$$

\section{THEN}

raise $k$ slightly

\section{ELSE}

lower $k$ slightly

FOR $(i=0$ to $3, i++)$

span $\sim \operatorname{span}^{*}$

\section{CONCLUSION AND SCOPE OF FUTURE RESEARCH}

The $h$ GA used by Bhattacharya and $\mathrm{Kumar}^{3}$ to solve the stochastic optimisation problem of minimising the option tracking error is found to be particularly susceptible to getting stuck in local optima especially when the return on the risky assets underlying the best-of option have temporally unstable correlation and volatility.

In this paper, we have proposed an extension of that $h$ GA model by incorporating a simple FLC that can make the model computationally more robust and thus, if adequately implemented, could become a potential decision support tool for portfolio managers. The actual computational implementation of an FLC-based $h \mathrm{GA}$ then becomes the immediate step to advance in the direction we have shown. Of course, a further obvious extension could be the ability of the model to accommodate polymorphisms that requires the model to be based on a diploid genetic algorithm (dGA) instead of an $h \mathrm{GA}$.

\section{References}

1 Black, F. and Scholes, M. (1973) 'The Pricing of Options and Corporate Liabilities', Journal of Political Economy, Vol. 81, No. 3, pp. 637-654. 
2 Johnson, H. (1987) 'Options on the Maximum and Minimum of Several Assets', Journal of Financial and Quantitative Analysis, Vol. 22, No. 3, pp. 277-283.

3 Bhattacharya, S. and Kumar, K. (2007) 'Computational Exploration of the Biological Basis of Black-Scholes Expected Utility Function', Journal of Applied Mathematics and Decision Sciences, Vol. 2007, Article ID 39460, doi:10.1155/2007/39460.

4 Robson, A.J. (1968) 'A Biological Basis for Expected and Non-expected Utility', Journal of Economic Theory, Vol. 68, pp. 397-424.

5 Robson, A.J. (2001) 'Why Would Nature Give Individuals Utility Functions', Journal of Political Economy, Vol. 4, pp. 900-914.
6 Becker, G.S. (1976) 'Altruism, Egoism and Genetic Fitness: Economics and Socio- biology', Journal of Economic Literature, Vol. 14, pp. 817-826.

7 Stulz, R. (1982) 'Option on the Minimum or Maximum of Two Assets', Journal of Financial Economics, Vol. 10, pp. 161-185.

8 Xu, H.U. and Vukovich, G. (1993) 'A Fuzzy Genetic Algorithm with Effective Search and Optimization', Proceedings of 1993 International Joint Conference on Neural Networks, Nagoya Congress Center, Japan, pp. 2967-2970.

9 Herrera, F., Herrera-Viedma, E., Lozano, M. and Verdegay, J.L. (1994) 'Fuzzy Tools to Improve Genetic Algorithms', Proceedings of the Second European Congress on Intelligent Techniques and Soft Computing, Aachen, Germany, pp. 1532-1539. 